15.1;07.2

\title{
Нелинейно-регрессионный алгоритм обработки сигналов полупроводниковых химических сенсоров, обеспечивающий селективное детектирование примесей в искусственном воздухе
}

\author{
(C) В.В. Чистяков, С.А. Казаков, М.А. Гревцев, С.М. Соловьев \\ Физико-технический институт им. А.Ф. Иоффе РАН, Санкт-Петербург, Россия \\ E-mail: v.chistyakov@mail.ioffe.ru
}

Поступило в Редакцию 28 сентября 2020 г.

В окончательной редакции 19 ноября 2020 г

Принято к публикации 1 декабря 2020 г.

Разработан новый способ обработки сигнала изменения электропроводности $\Delta \sigma$ при температурной $(T)$ модуляции химического сенсора для селективного определения в воздухе следовых концентраций аммиака, ацетона, н-гексана, пропана, толуола и других примесей. Способ заключается в том, что в диапазоне прецизионно устанавливаемых концентраций $C$ каждой из примесей $Y$ сигнал $\Delta \sigma$ как функция обратной температуры $z=10^{3} / T$ интерполируется при помощи нелинейной регрессии набором параметризованных функций $F_{i}\left(z, A_{i}, b_{i}, c_{i}, \ldots\right), i=1-4$, и строятся зависимости для главных (концентрационных) параметров $A_{i Y}(C)$, определяющие так называемый портрет селективности $Y$. Вписываясь в него, аналогичные величины у детектируемой примеси $X$ подтверждают ее тождественность с $Y$, а общая абсцисса всех точек пересечения линий уровня $A_{i X}$ с кривыми $A_{i Y}(C)$ определяет численное значение и единицу измерения концентрации $C_{X}$.

Ключевые слова: химический сенсор, температурная модуляция, селективность, нелинейная регрессия, концентрация, углеводороды, аммиак, ацетон.

DOI: 10.21883/PJTF.2021.06.50751.18564

Ввиду высокой чувствительности электропроводности тонких пленок поликристаллических $n$-полупроводников $\left(\mathrm{ZnO}, \mathrm{SnO}_{2}, \mathrm{SmS}\right.$ и др.) к хемосорбции из газовой среды на разветвленную поверхность как донорных $\left(\mathrm{H}_{2}, \mathrm{CH}_{4}\right.$, $\mathrm{CO}, \mathrm{C}_{2} \mathrm{H}_{6}, \mathrm{NH}_{3}$ и т.д.), так и акцепторных молекул $\left(\mathrm{O}_{2}, \mathrm{O}_{3}\right)$ давно утвердилось представление о таких пленках как о перспективных материалах для чувствительных элементов химических сенсоров - приборов искусственного обоняния [1]. При этом проблема различения (селекции) различных веществ традиционно решается при помощи так называемой термо- или температурной модуляции, когда рабочая температура $T[\mathrm{~K}]$ сенсора в среде изменяется в интервале $\left(T_{\text {low }}, T_{\text {up }}\right)$ монотонно либо циклическим образом [2-4].

В силу активационного характера хемосорбционных процессов температурная развертка должна давать различную кинетику прироста числа зонных носителей (электронов) и, следовательно, изменения во времени $t$ проводимости $\Delta \sigma(t)$ для частиц с разной энергетической структурой электронных оболочек, т. е. химией (см., например, [4], с. 1643).

Математическим инструментом анализа здесь служит дискретное преобразование Фурье (быстрое преобразование Фурье) [2] временно́го ряда сигнала $\Delta \sigma\left(t_{i}\right)$, $i=1-2^{N}$ при циклической термомодуляции с некоторым периодом $\tau$. При этом действительные части $\left(R_{k}, k=0,1,2, \ldots\right)$ получаемых коэффициентов Фурье связывают с высотой приповерхностного потенциального барьера, а мнимые $\left(I_{k}, k=1,2, \ldots\right)-$ с величиной собственно адсорбции на поверхности [4]. Методика, однако, до сих пор не реализована в виде универсаль- ного, надежного и простого компьютерного алгоритма распознавания примеси в анализируемой среде.

В настоящей работе предлагается иной подход, базирующийся на статистическом моделировании вышеозначенного сигнала. Подход дает контролируемые, объективные и в отличие от коэффициентов Фурье устойчивые к различным факторам интервальные, а не точечные оценки калибровочных характеристик. Опирающийся на аксиомы, выводы и формулы математической статистики, подход дозволяет тем самым применение базирующихся на ней теории надежности, теории принятия решений и вообще искусственного интеллекта. Поэтому он может быть реализован в виде ясного алгоритма с использованием коммерческого программного обеспечения и затем воплощен в реальных сенсорных системах c передачей сигнала через Bluetooth или Wi-Fi на сервер автоматической обработки, анализа и принятия решений.

Метод строится на соображениях, что сигналы рассматриваемого сенсора от разных адсорбатов (рис. 1) при температурной развертке („пиле“) несут в себе различия, выражающиеся как явно - в положении особых точек (максимумов, минимумов, перегибов) (рис. 1,a), так и неявно, кумулятивным образом, - во всех остальных уровнях временно́го/температурного ряда $\Delta \sigma\left(t_{k} / t_{k}^{0}\right)$, $k=1, \ldots, N=400 \ldots 800$.

Прежде всего для всех адсорбатов группы выбирают один и тот же график нагрева. Аргументом сигнала $\Delta \sigma(z)$ формально служит величина $z=10^{3} / T\left[\mathrm{~K}^{-1}\right]$ (рис. $1, b)$. 

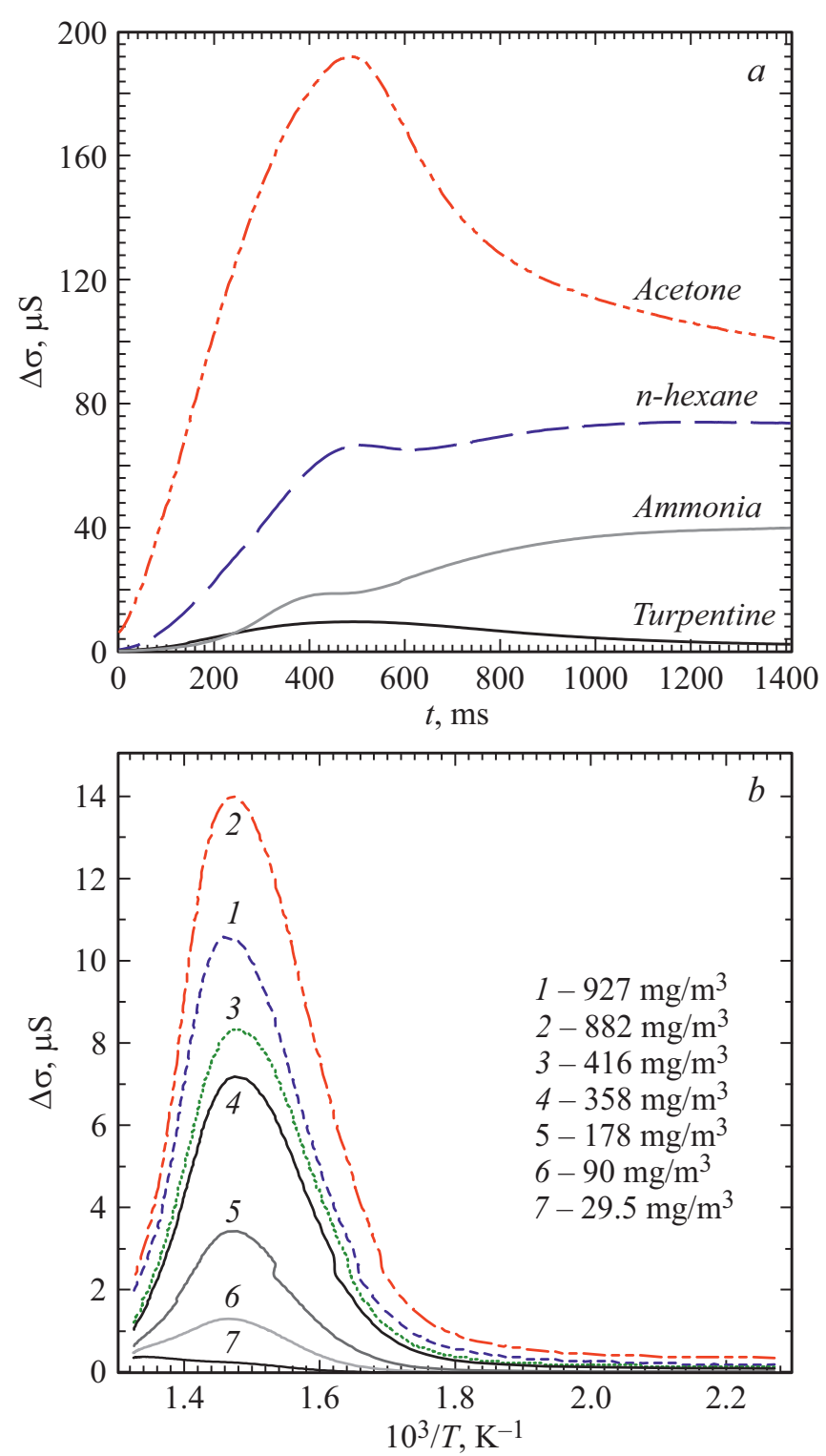

Рис. 1. Отклики прироста $\Delta \sigma$ электропроводности сенсора. $a$ - для одинаковой $\left(\sim 400 \mathrm{mg} / \mathrm{m}^{3}\right)$ концентрации разных веществ относительно времени экспозиции $t ; b$ - для различных концентраций паров сольвента относительно величины $z=10^{3} / T$.

Определяемые исходя из отклика величины должны обладать свойством робастности, т.е. устойчивости к 1) замене на эквивалентный набор данных, 2) одиночным выбросам (своеобразным дискретным „ $\delta$-функциям“, искажающим весь спектр Фурье) и 3 ) шумам сигнала. В качестве таковых выбирались значимо оцениваемые параметры формальной нелинейной по параметрам и объясняющей переменной регрессии сигнала $\Delta \sigma(z)$ с использованием ряда дискриминирующих функций $F_{n}(z)$ :

$$
F_{1}(z)=\frac{0.01 A_{1}}{z^{4}+b_{1} z^{3}+c_{1} z^{2}}
$$

$$
\begin{gathered}
F_{2}(z)=\frac{0.01 A_{2}}{z^{3}+b_{2} z^{2}+c_{2} z}, \\
F_{3}(z)=\frac{0.01 A_{3}}{z^{4}+b_{3} z^{3}+c_{3} z^{2}+d_{3} z}, \\
F_{4}(z)=\frac{0.0001 A_{4}}{z^{4}+b_{4} z^{3}+c_{4} z^{2}+d_{4} z+h_{4}}, \\
F_{5}(z)=\frac{0.01 A_{5}}{b_{5} z^{4}+c_{3} z^{3}+d_{5} z^{2}+h_{5} z+1}
\end{gathered}
$$

(множители 0.01/0.0001 использованы для более точной оценки малых значений главных параметров $A_{i}$ - центров соответствующих доверительных интервалов (ДИ) на доверительном уровне/вероятности $P=0.95)$.

В идеале все величины $A_{i}$ должны расти с концентрацией $C$. Но у одних монотонность утрачивается в силу немонотонности размаха отклика $\Delta \sigma$ на пары жидкостей, у других - из-за неизбежных флуктуаций получаемых оценок или иных причин.

Необходимо выполнение следующих требований к зависимостям $A_{i}(C)$ : 1) положительно определенность;
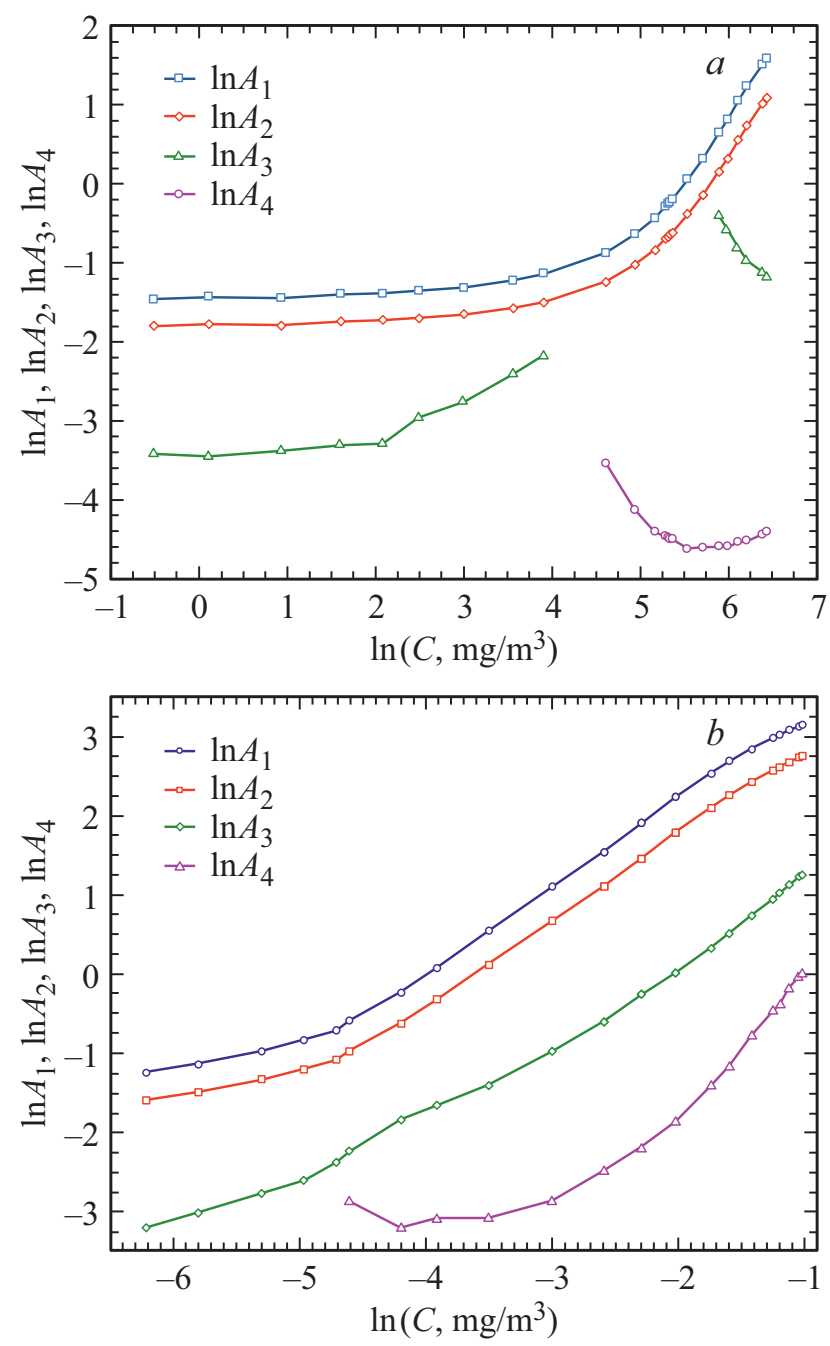

Рис. 2. Портреты селективности (gauge portraits) различных веществ в искусственном воздухе для сенсора на основе $\mathrm{SnO}_{2}$. $a$ - аммиак, $b-$ пропан. 
Таблица 1. Результаты оценки по формуле (1) параметров сигнала $\Delta \sigma / \mu \mathrm{S} \mathrm{SnO}{ }_{2}$-сенсора на концентрацию $C=951 \mathrm{mg} / \mathrm{m}^{3}$ толуола (доверительный уровень $P=0.95(95 \%)$ )

\begin{tabular}{c|r|c|c|r|r}
\hline \multirow{2}{*}{ Параметр } & \multicolumn{5}{|c}{$a d j-R^{2}=0.73335699$} \\
\cline { 2 - 6 } & Оценка & Стандартная ошибка & Критерий Стьюдента $t$ & Нижняя граница & Верхняя граница \\
\hline$A_{1}$ & 7.05398 & 0.253780 & 27.80 & 6.55546 & 7.55251 \\
$b_{1}$ & -3.08519 & 0.001573 & -1961.59 & -3.08827 & -3.08210 \\
$c_{1}$ & 2.38329 & 0.002524 & 944.40 & 2.37833 & 2.38824
\end{tabular}

Таблица 2. Результаты оценки по формуле (1) параметров сигнала $\Delta \sigma / \mu \mathrm{S} \mathrm{SnO}{ }_{2}$-сенсора на концентрацию $C=45 \mathrm{mg} / \mathrm{m}^{3}$ толуола (доверительный уровень $P=0.95(95 \%)$ )

\begin{tabular}{c|r|c|c|r|r}
\hline \multirow{2}{*}{ Параметр } & \multicolumn{5}{|c}{$a d j-R^{2}=0.99579415$} \\
\cline { 2 - 6 } & Оценка & Стандартная ошибка & Критерий Стьюдента $t$ & Нижняя граница & Верхняя граница \\
\hline$A_{1}$ & 0.10740 & 0.002137 & 50.27 & 0.10320 & 0.11160 \\
$b$ & -2.93652 & 0.001519 & -1933.04 & -2.93950 & -2.93354 \\
$c_{1}$ & 2.16033 & 0.002168 & 996.48 & 2.15607 & 2.16458
\end{tabular}

2) визуально непрерывность; 3) среди них обязательно должны быть монотонные в рабочем диапазоне концентраций или обладающие ярко выраженным растущим трендом и при этом 4) в координатах $\ln A_{i}-\ln C$ имеющие широкий участок крутого наклона.

В силу робастности значимых регрессионных оценок вообще фактически отпадают строгие требования на интервал температурной развертки $\Delta T$, тем более что границы диапазона регрессора $z$ слабо чувствительны к разумным изменениям уровней температурной „пилы“: на $10-20 \mathrm{~K}$ для нижнего $T_{\text {low }}$ и на $20-30 \mathrm{~K}$ для верхнего $T_{u p}=T_{\text {low }}+\Delta T$.

По структуре дискриминирующих функций (1)-(5) главные параметры $A_{i}(C)$ должны быстро меняться с концентрацией $C$ в противоположность медленно меняющимся $b_{i}(C), c_{i}(C), d_{i}(C), h_{i}(C)$, играющим вспомогательную идентифицирующую роль.

Кроме того, такую же роль могут играть также исправленный коэффициент детерминации $a d j-R^{2}$, показывающий в какой мере изменение сигнала $\Delta \sigma$ обусловлено изменением регрессора $z=10^{3} / T$, и величины критерия Стьюдента оценок $t\left(A_{i}\right), t\left(b_{i}\right), t\left(c_{i}\right), \ldots$, т. е. отношение сигнал/шум для всех оцениваемых параметров.

Основная идея метода заключается в том, что для каждой примеси из некоторой группы (аммиак, н-гексан, этанол, ацетон, сольвент, толуол и пр.) вышеозначенным образом оцениваются и строятся калибровочные кривые $A_{i}(C), i=1-4$ или 5, тем самым для каждого вещества формируется уникальный портрет селективности. Такой же набор $\left\{A_{i X}, i=1-4\right.$ или 5\} также путем регрессии по формулам (1)-(5) находится и для неизвестной концентрации неизвестной же примеси $X$. Если он (набор) вписывается в портрет селективности некоторого вещества $Y$, т.е. все горизонтальные линии $A_{i X}$ пересекаются со всеми соответствующими кривыми $A_{i Y}(C)$ на портрете $Y$ в точках с равными (в идеале) абсциссами $C^{*}$, то детектируемая примесь и есть $Y$, а $C^{*}-$ ее концентрация в единицах портрета $Y$.

Параметры оценивались опцией Nonlinear Estimation продукта StatSoft Statistica Release 12 (Trial, см. [5]), высокая оценка эффективности которого для обработки данных физических экспериментов дана в [6]. При этом минимизировалась стандартная сумма квадратов отклонений (loss function), а в качестве вычислительных алгоритмов служили методы Гаусса-Ньютона и Левенберга-Марквардта [5].

Начальные значения оцениваемых параметров, как правило, полагались нулевыми, но их можно получить из линейной по коэффициентам множественной регрессии для обратной величины сигнала $1 / \Delta \sigma[\mathrm{M} \Omega]$ в соответствии с модельными зависимостями $\frac{1}{F_{i}(z)}(1)-(5)$ $\left(\frac{1}{F_{i}(z)}=\alpha z^{4}+\beta z^{3}+\gamma z^{2}\right)$ с последующим пересчетом найденных коэффициентов.

В работе анализировались массивы данных по изменению сигнала электропроводности при температурной развертке от $\sim 430$ до $\sim 750 \mathrm{~K}$ примерно за время $\Delta \tau \sim 1.4 \mathrm{~s}$, полученные для тонких пленок poly- $\mathrm{SnO}_{2}(\mathrm{CuO})$, изготавливаемых по золь-гельтехнологии [1].

Функции (1)-(5) описывали экспериментальные данные при малых концентрациях с высоким коэффициентом $a d j-R^{2}=0.99 \ldots 0.999$, но с ростом $C$ величины $a d j-R^{2}$, как правило, монотонно плавно убывали до 0.73-0.80. При этом критерии Стьюдента оставались на высоком уровне: для главных параметров $t\left(A_{i}\right)$ порядка 15-150, для остальных коэффициентов $t\left(b_{i}\right), t\left(c_{i}\right)$, $t\left(d_{i}\right), t\left(h_{i}\right)-200-2000$ (табл. 1,2$)$.

Параметр считался оцененным значимо на уровне $P=0.95 \quad(\alpha=1-P=0.05)$, только если на таком 

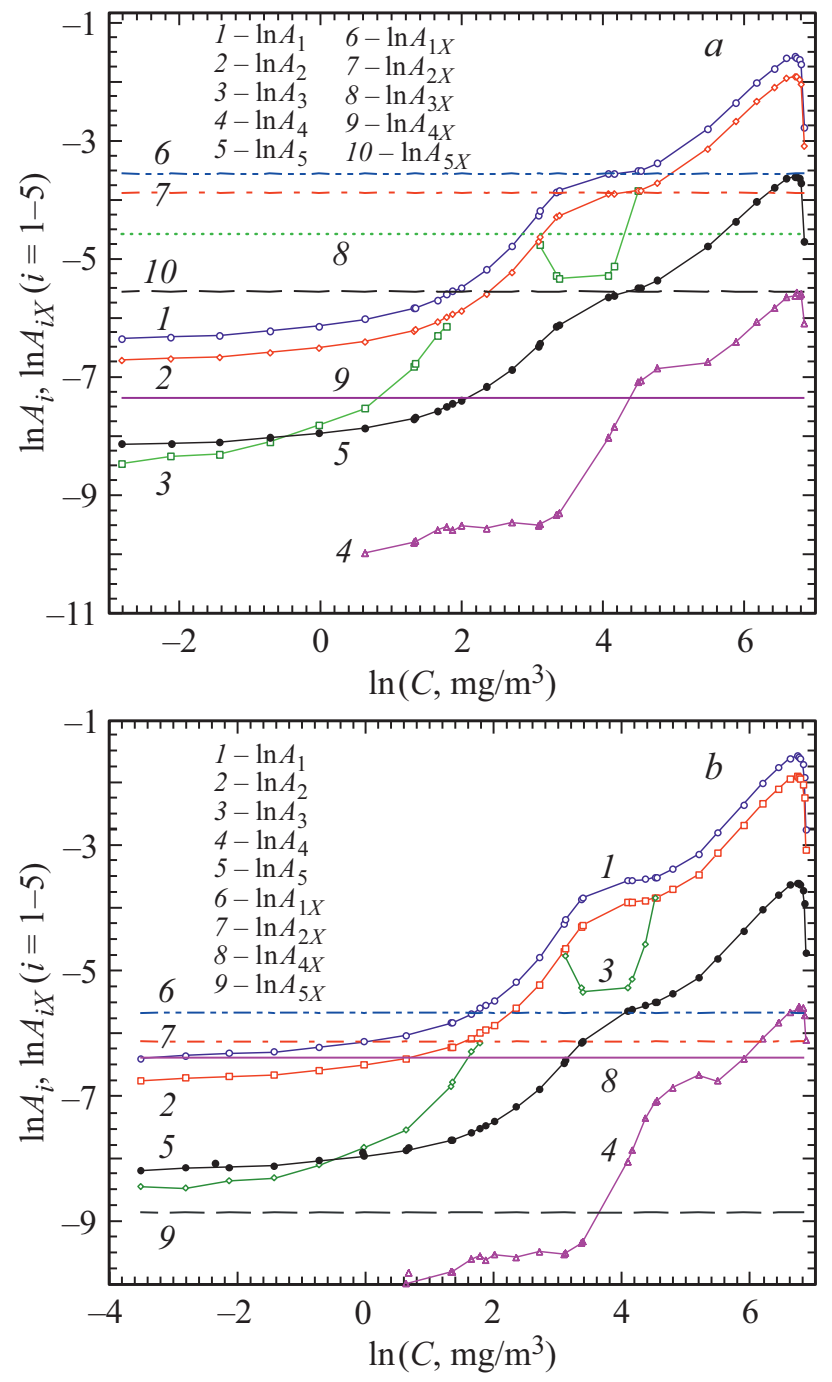

Рис. 3. Идентификация сольвент-не сольвент при помощи портрета селективности. $a-$ подтверждение в концентрации $72.2-79.8 \mathrm{mg} / \mathrm{m}^{3}$ (все линии уровня $\ln A_{i X}$ главных параметров для примеси $X$ пересекаются с соответствующими кривыми $\ln A_{i}(C)$ для сольвента в точках с одинаковыми абсциссами); $b$ - неподтверждение (абсциссы точек разные, для линии $\ln A_{5 X}$ пересечения нет вообще, величина $\ln A_{3 X}$ значимо не оценена).

уровне была значима вся их совокупность. Вследствие же незначимости на кривых $A_{3}(C)$ и $A_{4}(C)$ имели место пробелы. Параметры $A_{1}(C), A_{2}(C)$ и $A_{5}(C)$ значимо оценивались у всех веществ и при всех $C\left[\mathrm{mg} / \mathrm{m}^{3}\right.$, vol.\%], и именно их линии служили калибровочными кривыми.

Были получены различающиеся по виду наборы регулярных зависимостей $\ln A_{i}(\ln C)$ (портреты селективности) для следующих веществ в искусственном воздухе: аммиака, ацетона, н-гексана, пропана, сольвента и др. (рис. 2, 3); затем они использовались для безошибочной и точной идентификации газов из данного списка.

Пример такой идентификации паров неизвестного газа $X$ (со следующим ДИ $A_{i}: A_{1 X}$ low $=2.81870<A_{1 X}$ $<A_{1 X \text { up }}=2.90253, \ldots, A_{5 X \text { low }}=0.36973<A_{5 X}<A_{5 X \text { up }}$
$=0.39618)$, оказавшегося сольвентом в концентрации $\sim 78 \mathrm{mg} / \mathrm{m}^{3}$, схематично показан на рис. 3, $a$. Здесь горизонтальные линии центров ДИ $A_{i X}$ для газа $X$ пересекаются с кривыми портрета $Y$ (сольвент) в точках с визуально одинаковыми абсциссами $C^{*}$. В спорных случаях необходимо будет оперировать уже интервалами концентраций $C$, границы которых для каждой точки пересечения $(i)$ суть графические релевантные решения „уравнений“ $A_{i Y}$ ир $(C)=A_{i X}$ low и $A_{i Y}$ low $(C)=A_{i X \text { up. }}$ В том случае, если пересечение всех таких интервалов не есть пустое множество, детектируемая примесь соответствует портрету.

На рис. $3, b$ представлен обратный случай отрицательной идентификации неизвестного газа $X^{\prime}$ (толуола). Оба примера свидетельствуют о простоте и эффективности нелинейно-регрессионного метода. И даже немонотонность откликов сенсора по концентрации (рис. 3, $a$ ) не является здесь серьезной помехой для идентификации и измерения.

Таким образом, разработанный метод позволяет уверенно идентифицировать донорные микропримеси в искусственном воздухе. Его возможности и ресурсы далеко не исчерпаны как в плане оптимизации по параметрам развертки, так и в плане применения искусственного интеллекта [7].

Метод уже сейчас может быть обобщен на детектирование двух примесей $(X 1, X 2)$, например пропана и паров воды. На предварительно построенных 3D-портретах $A_{i}\left(C_{1}, C_{2}\right)$ линии уровня параметров $A_{i X 1, X 2}$, будучи спроецированы на плоскость концентраций $\left(C_{X 1}, C_{X 2}\right)$, пересекутся там в одной точке, которая и определит состав двойной примеси.

\section{Финансирование работы}

Исследование выполнено в рамках гранта Российского фонда фундаментальных исследований № 18-03-00660.

\section{Конфликт интересов}

Авторы заявляют, что у них нет конфликта интересов.

\section{Список литературы}

[1] K.S. Shalini Devi, A. Anantharamakrishnan, U. Maheswari Krishnan, J. Yakhmi, in Smart sensors for environmental and medical applications (IEEE, Inc., 2020), p. 103-127. DOI: $10.1002 / 9781119587422$

[2] S. Nakata, S. Akakabe, M. Nakasuji, K. Yoshikawa, Anal. Chem., 68, 2067 (1996).

[3] A. Heilig, N. Bârsan, U. Weimar, M. Scheizer-Berberich, J.W. Gardner, W. Göpel, Sensors Actuators B, 43, 46 (1997).

[4] S. Nakata, T. Hashimoto, H. Okunishi, Analyst, 127, 1642 (2002). DOI: $10.1039 / \mathrm{b} 208295 \mathrm{k}$

[5] https://www.statsoft.de

[6] В.В. Чистяков, Физическое образование в вузах, 21 (1), 120 (2015).

[7] P. Gwiźdź, A. Brudniak, K. Zakrewska, Metrol. Meas. Syst., XXII (1), 3 (2015). DOI: 10.1515/mms-2015-0008 